\title{
Monitoring for the Distribution of Phytoplankton along the Hypersaline Bardawil Lagoon, in Northern Sinai, Egypt
}

\author{
Jelan Mofeed \\ Aquatic Environment Department, Faculty of Fish Resources, Suez University,Egypt.
}

\begin{abstract}
Bardawil Lagoon is a source of wildlife and high-quality fish, so, it is imperative that it should be subject to continuous monitoring of both water quality and the phytoplankton composition. Samples were collected from ten sites representing different habitats of the lagoon, from summer 2017 to spring 2018. The water properties were determined; noticeable low concentrations of dissolved inorganic nutrients were recorded. A total of 106 species belonging to six groups were recorded, among them Bacillariophyta (69 species) and Dinophyta (26 species). The obtained results clarify that Bacillariophytes prevailed in the eastern sites of Bardawil; on the contrary, Dinophytes occupied the sovereignty in the western sites of the lagoon. The most abundant Bacillariophytes were Licmophora gracilis, Gyrosigma acuminatum, Fragilaria construens, and Nitzschia sigmoidea; which formed more than $50 \%$ of total abounded diatoms. While, Protoperidinium leonis, Prorocentrum gracile and Protoperidinium granii were the dominant Dinophytes, giving it maximum at El-Rewak. From reviewing previous studies on the lagoon, it becomes clear that the phytoplankton composition varied widely from previously recorded, including the dominant species and their rates of sovereignty, which reflected economically on its fish productivity. Besides, the water quality in Bardawil fluctuated from slightly-polluted to moderately-polluted according to the diversity index, as a result of the recent, noticeable increase in human activities, especially fishing, which will be in turn reflected in the environment. Therefore, continuous follow-up through ecological assessment and monitoring studies of Bardawil became an urgent necessity.
\end{abstract}

Keywords: Bardawil lagoon, phytoplankton distribution, water quality.

\section{INTRODUCTION}

Bardawil Lagoon is a large shallow hyper-saline coastal lagoon, existed in the middle Mediterranean coast of Sinai Peninsula, Egypt. This lagoon constituted about $22 \%$ of the total northern lagoons' area, where it has an area of about $685 \mathrm{~km}^{2}$ (Khalil and Shaltout, 2006). Seawater enters the lagoon through two artificial tidal inlets (Boughaz I and II) opened periodically by dredging. During Israeli occupation to Sinai in 1967 , both inlets were closed by 1970 . Thus, the salinity of the lagoon had undergone a drastic increase that reached up to $120 \%$ (Pisanty, 1980). The inlets were reopened from 1972 until 1978, and then salinity decreased to $38.5 \%$ at the Boughaze area and it was $74.5 \%$ at the most western part of the lagoon.

Bardawil Lagoon reported as oligotrophic to mesotrophic ecosystem (Touliabah et al., 2002). Where, it is the least polluted lagoon because it did not receive any drainage canals. Therefore, it is an important source of good quality, local and economic fishes in North Sinai, and it plays an essential role in the fish production in Egypt and most of its catch is exported. Bardawil Lagoon produces over 2,500 tons annually; where it characterized by very economically important species of fishes such as sea bass, sea bream, sole, grey mullet, eel, meager and white grouper (GAFRD, 2012). Taking into consideration, fishing is stopped from January to May, in order to allow fish stocks to recuperate. Moreover, it is an Important Bird Area by BirdLife International, where, it is an essential stop and staging site for massive numbers of migrants passing through Zaranik protected area especially in autumn months, besides its important for wintering water-birds e.g. Phalacrocorax carbo and Phoenicopt- erus rubber (Khalil and Shaltout, 2006). Ali et al., (2006) reported that, two species of the most common migrants birds namely; Sterna albifrons and Charadrius alexandrinus, breed in the immediate vicinity of the lagoon with internationally important numbers.

Algae are an essential partner in the aquatic ecosystem (Mofeed, 2015a). Phytoplankton composes the base of the food chain in the aquatic environment where it forms the main primary producers (ShaabanDessouki et al., 2004; Mofeed and Mosleh, 2013). Phytoplankton biomass in Bardawil Lagoon is generally low but during summer and autumn, it increases due to dinoflagellates and diatoms dominancy. Both blue-green and green algae are comparatively insignificant in the phytoplankton composition. This coastal lagoon is currently oligotrophic to mesotrophic ecosystem (Touliabah et al., 2002). During recent years, the lagoon suffered from many problems, due to the expansion of human activities and tourism around the lagoon and due to lack of systematic follow-up to the continuous dredging of the two artificial inlets (Boughaz I and II). In addition to the increase in many unauthorized human activities around the lagoon. These problems might cause an increase in salinity and pollutant concentration, and then lead to environmental degradation, which significantly disturbed the phytoplankton composition (the main producer of the food chain) and consequently, it constitutes a threat to the ecosystem as a whole in this virgin lagoon, which contains one of the most important natural reserves in the world which considered a shelter to many of the migratory organisms, among them are threatened species (El-Sheekh et al., 2019). Also, this will be reflected in the productivity of the lake, which is of 
great importance in the national economy, as the lake produces large quantities of fish of high quality and desirable for export. Where the expensive species is $77.05 \%$ of its total fish production, but due to the illegal human activities, the total loss of biomass was 4723 tons only one fishing season (El-Aiatt et al., 2019.

Therefore, Bardawil Lagoon still in need of sustainable monitoring studies to provide a database for water quality status and to maintain the purity and health of this lagoon and put a proper management strategy. The main objectives of the present study are to evaluate the water quality characteristics and to determine the phytoplankton community composition and its distributional pattern with the different ecological variables in Bardawil lagoon. This data can be used as a database that will be benefiting the subsequent monitoring studies.

\section{MATERIALS AND METHOD}

\section{Study area}

In the North of Sinai Peninsula, Egypt and between longitudes $320^{\circ} 40^{\prime}$ and $330^{\circ} 30^{\prime \prime} \mathrm{E}$ and latitudes $310^{\circ} 03^{\prime}$ and $310^{\circ} 141^{\prime} \mathrm{N}$, a large Lagoon is known as Bardawil Lagoon situated. The southern side of the lagoon surrounded by desert and sand dunes, while the northern border by the Mediterranean Sea. Based on satellite image interpretations and GIS techniques, the lagoon extends for about $80 \mathrm{~km}$ along on the northern coast of Sinai between El-Qantara and El-Arish Cities.
Its maximum width is about $20.5 \mathrm{~km}$ and the lake is very shallow, with a mean depth of around $1.5 \mathrm{~m}$ and a maximum of $7.5 \mathrm{~m}$. The maximum depth in Boughaz (II) is $5.75 \mathrm{~m}$ due to dredging. The surface area is about $685 \mathrm{~km} 2$. The Lagoon has an elliptical shape, separated from the Mediterranean Sea by a curving sand barrier with a width between 300 and $2000 \mathrm{~m}$. There are two artificial inlets (Boughazes I and II) connect the Lagoon to the sea (Negm et al., 2019) and two small natural eastern inlets (Boughaz Zaranik and Abo Salah) which are now occasionally closed by silting, that have been established to decrease the salinity through the exchange of water with the Mediterranean Sea. The main water supply to the Lagoon comes from the Mediterranean Sea, which flows constantly, mainly through the first two openings (Khalil and Shaltout, 2006). The lagoon characterized by arid climate and low precipitation rate (5-10 mm.year ${ }^{-1}$ ) only during the winter months, accompanied by high evaporation rates without streams flow inside the lagoon (Zalat et al., 2019)

\section{Samples Collection}

As illustrated in the Bardawil Lagoon map (Figure 1), ten sites were selected for sampling, to cover the whole area and represent all the habitats of the Lagoon (Table 1). Surface water samples were collected seasonally from summer 2017 to spring 2018 using the Ruttner Water Sampler bottle (capacity of 2L).

Table (1): Names of the selected sites in Bardawil Lagoon, with their latitudes and longitude.

\begin{tabular}{cccccccc}
\hline \hline Site No. & Site Name & Longitude & Latitude & Site No. & Site Name & Longitude & Latitude \\
\hline 1 & Rabaa & $32^{\circ} 44^{\prime} 33^{\prime \prime}$ & $31^{\circ} 03^{\prime} 24^{\prime \prime}$ & 6 & Masqut-Eplis & $33^{\circ} 09^{\prime} 20^{\prime \prime}$ & $31^{\circ} 11^{\prime} 47^{\prime \prime}$ \\
2 & El-Nasser & $32^{\circ} 49^{\prime} 17^{\prime \prime}$ & $31^{\circ} 04^{\prime} 55^{\prime \prime}$ & 7 & Boughaz II & $33^{\circ} 15^{\prime} 41^{\prime \prime}$ & $31^{\circ} 12^{\prime} 15^{\prime \prime}$ \\
3 & Boughaz I & $32^{\circ} 55^{\prime} 47^{\prime \prime}$ & $31^{\circ} 08^{\prime} 01^{\prime \prime}$ & 8 & El- Zaranik & $33^{\circ} 16^{\prime} 51^{\prime \prime}$ & $31^{\circ} 07^{\prime} 03^{\prime \prime}$ \\
4 & El-Rewak & $33^{\circ} 00^{\prime} 02^{\prime \prime}$ & $31^{\circ} 03^{\prime} 50^{\prime \prime}$ & 9 & El-Rodh & $33^{\circ} 15^{\prime} 03^{\prime \prime}$ & $31^{\circ} 05^{\prime} 58^{\prime \prime}$ \\
5 & El-Gals & $33^{\circ} 05^{\prime} 54^{\prime \prime}$ & $31^{\circ} 11^{\prime} 26^{\prime \prime}$ & 10 & El-Telol & $33^{\circ} 13^{\prime} 36^{\prime \prime}$ & $31^{\circ} 04^{\prime} 37^{\prime \prime}$ \\
\hline \hline
\end{tabular}

\section{Chemical analysis}

Salinity, $\mathrm{pH}$ and dissolved oxygen were measured in the field by using sing Hydrolab, Model (Multi-Set 430i WTW) according to APHA (1989). Water samples were filtered using GF/C microfiber filter paper to determine its chemical composition. Ammonia concentrations were determined colorimetrically by indophenol's method (Bremner and Shaw, 1955). According to Kampshake et al., (1967), by hydrazine reduction method, nitrates were estimated. Nitrites were determined using the colorimetric coupled method according to Barnes and Folkard (1951). While ortho-phosphate and silicate were determined after extraction in $0.5 \mathrm{M} \mathrm{NaOH}$ according to Hartikainen (1979) and Krausse et al., (1983) respectively. Moreover, heavy metals $(\mathrm{Mn}, \mathrm{Zn}, \mathrm{Cu}$, and $\mathrm{Pb}$ ) were estimated according to Ajaykumar et al., (2008).

\section{Phycological analysis}

One litter of each water sample was fixed by using Lugol solution before identification and enumeration by using the microscope at $15 \mathrm{X}$ eyepieces and $40 \mathrm{X}$ and $100 \mathrm{X}$ objective, to identify the algal taxa to the species level. The following references were used for phytoplankton identification; Prescott (1962, 1969 and 1982), Hendey (1964), Patrick and Reimer (1966), Foged (1978), Starmach (1968 and1983), Baker (1991), Yamagishi (1992).

\section{Diversity index}

The diversity of phytoplankton community was calculated according to Shannon and Weaver (1963).

\section{Statistical analysis}

One-way analysis of variance (ANOVA) used to define the significant variation in parameters (Anonymous, 1993). MVSP program, multidimensional analyses (Cluster analysis) classify similarity between the data. In addition, the abundance index performed using the MVSP program in order to determine the most common species during the study period (Legendre and Legendre, 1998). Pearson's 
correlation coefficients were achieved out by the statistical software SPSS (Version 14.0 for Windows). Multivariate analysis of Detrended correspondence analysis and (DCA) Canonical correspondence analysis (CCA) used to clarify the variation in phytoplankton assemblages structure and then related to environmental factors by using CANOCO V. 4.0 program (Ter Braak, 1988).

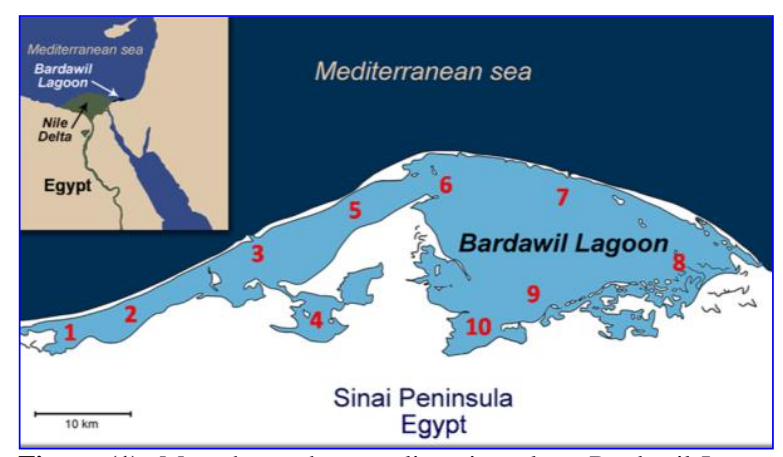

Figure (1): Map shows the sampling sites along Bardawil Lagoon, North Sinai, Egypt.

\section{RESULTS}

Inspection in Table (2) reflected the environmental situation of Bardawil Lagoon, where the mean value of the hydrogen ion concentration $(\mathrm{pH})$ fluctuated in a narrow range (from 8.55 to 8.25) during the investigation period giving its maximum value at Rabaa (site 1). In contrast, a significant wide range of variation in salinity recorded where it gave its maximum mean value $\left(60.8 \mathrm{~g} . \mathrm{L}^{-1}\right)$ at Rabaa followed by El-Telol, El-Nasser, and El-Rodh (55.2, 53.2 and $53.2 \mathrm{~g} . \mathrm{L}^{-1}$ respectively). It is noticeable that, generally the maximum salinity was recorded in the eastern and western edges of the lake, while the minimum values were obtained near both Boughaz I (from 37.9 to 41.3 g.L $\mathrm{L}^{-1}$ ) and Boughaz II (from 37.5 to 41.4 g.L $\mathrm{L}^{-1}$ ). Concerning correlation (Table 3 ) of salinity with the other chemical parameters, it gave remarkable high positive correlations with all parameters except orthophosphate (- 0.75) and dissolved oxygen (-0.59giving its maximum value at Rabaa (site 1). In contrast, a significant wide range of variation in salinity recorded where the maximum mean value was 60.8 g.L $\mathrm{L}^{-1}$. at Rabaa, followed by El-Telol, El-Nasser, and El-Rodh (55.2, 53.2 and 53.2 g. $\mathrm{L}^{-1}$ respectively). It is noticeable that, the maximum salinity was recorded in the eastern and western edges of the lake, while the minimum values were recorded near both Boughaz I (from 37.9 to 41.3 g.L ${ }^{-1}$ ) and Boughaz II (from 37.5 to 41.4 g.L ${ }^{-1}$ ). Concerning correlation (Table 3 ) of salinity with the other chemical parameters, it gave remarkable high positive correlations with all parameters except orthophosphate (- 0.75) and dissolved oxygen (-0.59).

The mean value of the measured dissolved oxygen (DO) was higher than $8 \mathrm{mg} . \mathrm{L}^{-1}$ at Boughaz II, Boughaz I, and El- Zaranik. Meanwhile, lower values were recorded in El-Rewak (4.2 - $\left.6.5 \mathrm{mg} . \mathrm{L}^{-1}\right)$, with a mean value of $5.4 \mathrm{mg} . \mathrm{L}^{-1}$. Paradoxically, El-Rewak showed the maximum biological oxygen demand (BOD) during the study period $\left(2.5-6.7 \mathrm{mg} . \mathrm{L}^{-1}\right)$ with a mean value of $4.6 \mathrm{mg} . \mathrm{L}^{-1}$. Whereas, the minimum BOD values were obtained at Masqut-Eplis $\left(2.0 \mathrm{mg} . \mathrm{L}^{-1}\right)$ followed by Boughaz II $\left(2.3 \mathrm{mg} . \mathrm{L}^{-1}\right)$. In this context, DO was negatively correlated with BOD (-0.84) and ammonia $(-0.79)$ at $P \leq 0.005$ (Table 3 ). In contrast, BOD positively correlated with ammonia (0.86).

However, among the recorded values of the inorganic nitrogen (nitrate, nitrite, and ammonia), the most prominent phenomena that the minimum values were recorded at El-Gals $\left(4.8,36.3\right.$ and $31 \mu \mathrm{g} . \mathrm{L}^{-1}$ for nitrite, nitrate, and ammonia respectively) followed by Boughaz II (5.2 and 38.1 $\mu \mathrm{g} . \mathrm{L}^{-1}$ for nitrite and nitrate respectively). It is clear from the cited results that, ortho-phosphate fluctuated between $48 \mu \mathrm{g}$. $\mathrm{L}^{-1}$ (at Boughaz I) and $32 \mu \mathrm{g} . \mathrm{L}^{-1}$ (at El-Telol) within Bardawil lagoon. However, silicate achieved its maximum (133 $\left.\mu \mathrm{g} . \mathrm{L}^{-1}\right)$ at El-Telol. A glance of Pearson correlation coefficient table, clarify that, orthophosphate showed a negative correlation with ammonia (-0.78) and BOD (-0.61). Anent the estimated heavy metals (Table 2) revealed that, the maximum concentrations of cobber $\left(25 \mu \mathrm{g} . \mathrm{L}^{-1}\right)$, zinc $\left(375 \mu \mathrm{g} . \mathrm{L}^{-1}\right)$, lead $\left(51 \mu \mathrm{g} . \mathrm{L}^{-1}\right)$ and manganese $\left(72 \mu \mathrm{g} . \mathrm{L}^{-1}\right)$ were recorded in El-Telol, while El-Rodh and Rabaa come in the second and third position with a significant gap.

Table. 4 clarify that, a total of 106 taxa of six algal groups (68 Bacillariophyta; 26 Dinophyta; 5 Chlorophyta; 4 Cyanophyta; 2 Euglenophyta and 1 Chrysophyta) were recorded within the studied sites along Bardawil Lagoon (Figure 2). As shown in table 5, the maximum total number of species was recorded at Boughaz I (61 species), followed by Boughaz II (59 species), and El- Zaranik (49 species). While, only 33 species were recorded at Rabaa. Concerning the number of species belonging to different algal groups within each site along Bardawil lagoon (Table. 5), a remarkable superiority of Bacillariophytes was observed, where it had the topmost number of species in all sites during the study period (Average: 26.2). The maximum number (41 species) of Bacillariophyta species was recorded at Boughaz I "site.3". In this context, Boughaz I also achieved the maximum number of species (17 species) belonging to Dinophyta and consequently the maximum total number of species (61 species). In general, Dinophyta occupies the second position after Bacillariophyta as number of species (Average: 13.3). Contrarily, Cyanophyta, Euglenophyta, Chlorophyta and even Chrysophyta represented by a limited number of species in all Bardawil Lagoon' sites during the entire period of study.

Meanwhile, considering the abundance of each algal group to the total abundance cover "as cell number" within the studied sites (Figure 3) revealed that, again Bacillariophyta dominated over the other groups followed by Dinophyta. In this context the maximum total cell number of Bacillariophyta $\left(304\right.$ cell X10 $\left.10^{4} \cdot \mathrm{L}^{-1}\right)$ was recorded at El-Rewak site, followed by El-Telol $\left(276\right.$ cell $\left.\mathrm{X} 10^{4} \cdot \mathrm{L}^{-1}\right)$ and Rabaa (264 cell X10 $\left.{ }^{4} \cdot \mathrm{L}^{-1}\right)$, 
Monitoring for Bardawil Lagoon

Table (2): The measured annual average of the physico-chemical parameters of water at the studied sites along Bardawil Lagoon.

\begin{tabular}{|c|c|c|c|c|c|c|c|c|c|c|c|c|}
\hline \multirow{2}{*}{$\begin{array}{l}\text { Measured } \\
\text { Parameters }\end{array}$} & \multirow[t]{2}{*}{$\dagger$} & \multicolumn{10}{|c|}{ Studied Sites } & \multirow{2}{*}{$\begin{array}{l}\text { Annual } \\
\text { average }\end{array}$} \\
\hline & & 1 & 2 & 3 & 4 & 5 & 6 & 7 & 8 & 9 & 10 & \\
\hline pH & $\begin{array}{l}\mathrm{R} \\
\mathrm{M}\end{array}$ & $\begin{array}{l}7.9-8.8 \\
8.55 \pm 0.24\end{array}$ & $\begin{array}{l}8.2-8.7 \\
8.48 \pm 0.74\end{array}$ & $\begin{array}{l}8.1-8.3 \\
8.25 \pm 0.14\end{array}$ & $\begin{array}{l}8.0-8.4 \\
8.31 \pm 0.22\end{array}$ & $\begin{array}{l}8.2-8.7 \\
8.54 \pm 0.62\end{array}$ & $\begin{array}{l}8.2-8.6 \\
8.45 \pm 0.72\end{array}$ & $\begin{array}{l}8.1-8.5 \\
8.47 \pm 0.38\end{array}$ & $\begin{array}{l}8.0-8.5 \\
8.26 \pm 0.25\end{array}$ & $\begin{array}{l}8.0-8.7 \\
8.31 \pm 0.47\end{array}$ & $\begin{array}{l}7.6-8.5 \\
8.42 \pm 0.34\end{array}$ & 8.40 \\
\hline $\begin{array}{l}\text { Salinity } \\
\left(\text { g. } L^{-1}\right)\end{array}$ & $\begin{array}{l}\mathrm{R} \\
\mathrm{M}\end{array}$ & $\begin{array}{l}50.3-72.7 \\
60.8 \pm 2.17\end{array}$ & $\begin{array}{l}45.5-60.3 \\
53.2 \pm 2.33\end{array}$ & $\begin{array}{l}37.9-41.3 \\
39.4 \pm 1.36\end{array}$ & $\begin{array}{l}40.2-54.7 \\
46.6 \pm 2.23\end{array}$ & $\begin{array}{l}43.5-53.0 \\
49.1 \pm 1.94\end{array}$ & $\begin{array}{l}43.0-51.5 \\
48.2 \pm 2.24\end{array}$ & $\begin{array}{l}37.5-41.4 \\
39.3 \pm 1.81\end{array}$ & $\begin{array}{l}42.2-55.4 \\
49.2 \pm 2.27\end{array}$ & $\begin{array}{l}45.3-63.2 \\
50.8 \pm 3.04\end{array}$ & $\begin{array}{l}45.3-64.5 \\
55.2 \pm 3.11\end{array}$ & 49.18 \\
\hline DO $\left(\mathrm{mg}^{-L^{-1}}\right)$ & $\begin{array}{l}\mathrm{R} \\
\mathrm{M}\end{array}$ & $\begin{array}{l}5.0-7.1 \\
6.0 \pm 0.14\end{array}$ & $\begin{array}{l}5.3-7.5 \\
6.5 \pm 0.41\end{array}$ & $\begin{array}{l}6.4-8.8 \\
8.1 \pm 0.04\end{array}$ & $\begin{array}{l}4.2-6.5 \\
5.4 \pm 0.21\end{array}$ & $\begin{array}{l}5.6-7.8 \\
6.7 \pm 0.34\end{array}$ & $\begin{array}{l}5.2-8.8 \\
7.8 \pm 0.42\end{array}$ & $\begin{array}{l}6.8-9.9 \\
8.5 \pm 0.27\end{array}$ & $\begin{array}{l}5.9-8.6 \\
8.1 \pm 0.18\end{array}$ & $\begin{array}{l}5.3-7.1 \\
6.6 \pm 0.19\end{array}$ & $\begin{array}{l}5.5-6.8 \\
6.0 \pm 0.23\end{array}$ & 6.97 \\
\hline BOD $\left(\mathrm{mg} \cdot \mathrm{L}^{-1}\right)$ & $\begin{array}{l}\mathrm{R} \\
\mathrm{M}\end{array}$ & $\begin{array}{l}3.0-7.1 \\
4.3 \pm 0.08\end{array}$ & $\begin{array}{l}1.9-4.8 \\
3.6 \pm 0.14\end{array}$ & $\begin{array}{l}1.6-4.0 \\
2.8 \pm 0.25\end{array}$ & $\begin{array}{l}2.5-6.7 \\
4.6 \pm 0.34\end{array}$ & $\begin{array}{l}1.7-4.4 \\
3.1 \pm 0.04\end{array}$ & $\begin{array}{l}1.3-4.5 \\
2.0 \pm 0.10\end{array}$ & $\begin{array}{l}1.5-3.8 \\
2.3 \pm 0.11\end{array}$ & $\begin{array}{l}1.6-4.6 \\
2.9 \pm 0.21\end{array}$ & $\begin{array}{l}2.6-4.8 \\
3.5 \pm 0.08\end{array}$ & $\begin{array}{l}2.0-5.9 \\
3.9 \pm 0.13\end{array}$ & 3.3 \\
\hline $\mathrm{NO}_{2}\left(\mu \mathrm{g} . \mathrm{L}^{-1}\right)$ & $\begin{array}{l}\mathrm{R} \\
\mathrm{M}\end{array}$ & $\begin{array}{l}0.0-10.0 \\
6.9 \pm 0.33\end{array}$ & $\begin{array}{l}2.2-15.0 \\
6.1 \pm 0.21\end{array}$ & $\begin{array}{l}0.0-17.0 \\
6.6 \pm 0.26\end{array}$ & $\begin{array}{l}0.0-14.8 \\
7.3 \pm 0.33\end{array}$ & $\begin{array}{l}0.0-14.9 \\
4.8 \pm 0.51\end{array}$ & $\begin{array}{l}0.0-12.4 \\
6.5 \pm 0.28\end{array}$ & $\begin{array}{l}0.0-15.5 \\
5.2 \pm 0.14\end{array}$ & $\begin{array}{l}0.0-11.9 \\
5.7 \pm 0.22\end{array}$ & $\begin{array}{l}0.0-15.3 \\
5.3 \pm 0.09\end{array}$ & $\begin{array}{l}5.5-20.2 \\
9.8 \pm 0.36\end{array}$ & 6.53 \\
\hline $\mathrm{NO}_{3}\left(\mu \mathrm{g} . \mathrm{L}^{-1}\right)$ & $\begin{array}{l}\mathrm{R} \\
\mathrm{M}\end{array}$ & $\begin{array}{l}10.8-68.2 \\
46.1 \pm 1.25\end{array}$ & $\begin{array}{l}13.6-59.8 \\
46.4 \pm 1.24\end{array}$ & $\begin{array}{l}20.3-64.5 \\
39.2 \pm 2.04\end{array}$ & $\begin{array}{l}16.2-74.3 \\
42.5 \pm 2.20\end{array}$ & $\begin{array}{l}17.6-51.8 \\
36.3 \pm 1.94\end{array}$ & $\begin{array}{l}17.9-68.4 \\
43.2 \pm 2.15\end{array}$ & $\begin{array}{l}16.2-58.3 \\
38.1 \pm 1.84\end{array}$ & $\begin{array}{l}21.1-74.6 \\
46.0 \pm 1.76\end{array}$ & $\begin{array}{l}14.4-72.3 \\
46.0 \pm 2.04\end{array}$ & $\begin{array}{l}16.7-79.2 \\
58.6 \pm 2.32\end{array}$ & 43.32 \\
\hline $\begin{array}{l}\mathbf{N H}_{4} \\
\left(\mu \mathrm{g} . \mathrm{L}^{-1}\right)\end{array}$ & $\begin{array}{l}\mathrm{R} \\
\mathrm{M}\end{array}$ & $\begin{array}{l}32-81 \\
66 \pm 1.24\end{array}$ & $\begin{array}{l}15-95 \\
44 \pm 1.28\end{array}$ & $\begin{array}{l}20-179 \\
40 \pm 2.37\end{array}$ & $\begin{array}{l}22-81 \\
56 \pm 1.94\end{array}$ & $\begin{array}{l}32-85 \\
31 \pm 1.84\end{array}$ & $\begin{array}{l}21-79 \\
50 \pm 2.15\end{array}$ & $\begin{array}{l}18-69 \\
34 \pm 0.99\end{array}$ & $\begin{array}{l}15-61 \\
44 \pm 1.27\end{array}$ & $\begin{array}{l}48-139 \\
54 \pm 2.20\end{array}$ & $\begin{array}{l}55-193 \\
96 \pm 3.14\end{array}$ & 51.9 \\
\hline $\begin{array}{l}\text { Ortho-P } \\
\left(\mu \mathrm{g} . \mathrm{L}^{-1}\right)\end{array}$ & $\begin{array}{l}\mathrm{R} \\
\mathrm{M}\end{array}$ & $\begin{array}{l}21-92 \\
38 \pm 0.97\end{array}$ & $\begin{array}{l}14-82 \\
40 \pm 1.60\end{array}$ & $\begin{array}{l}28-104 \\
48 \pm 1.55\end{array}$ & $\begin{array}{l}18-82 \\
40 \pm 0.94\end{array}$ & $\begin{array}{l}27-97 \\
36 \pm 0.86\end{array}$ & $\begin{array}{l}25-82 \\
39 \pm 1.31\end{array}$ & $\begin{array}{l}26-95 \\
46 \pm 2.04\end{array}$ & $\begin{array}{l}12-68 \\
38 \pm 1.23\end{array}$ & $\begin{array}{l}23-83 \\
40 \pm 1.70\end{array}$ & $\begin{array}{l}17-59 \\
32 \pm 0.94\end{array}$ & 39.7 \\
\hline $\mathrm{SiO}_{3}\left(\mu \mathrm{g} . \mathrm{L}^{-1}\right)$ & $\begin{array}{l}\mathrm{R} \\
\mathrm{M}\end{array}$ & $\begin{array}{l}39-140 \\
94 \pm 4.31\end{array}$ & $\begin{array}{l}62-145 \\
87 \pm 2.27\end{array}$ & $\begin{array}{l}29-139 \\
98 \pm 3.32\end{array}$ & $\begin{array}{l}54-207 \\
85 \pm 1.95\end{array}$ & $\begin{array}{l}25-85 \\
69 \pm 1.24\end{array}$ & $\begin{array}{l}61-93 \\
94 \pm 3.20\end{array}$ & $\begin{array}{l}61-84 \\
80 \pm 2.45\end{array}$ & $\begin{array}{l}32-132 \\
98 \pm 2.74\end{array}$ & $\begin{array}{l}48-137 \\
109 \pm 3.88\end{array}$ & $\begin{array}{l}58-218 \\
133 \pm 3.92\end{array}$ & 94.7 \\
\hline $\mathrm{Cu}\left(\mu \mathrm{g} . \mathrm{L}^{-1}\right)$ & $\begin{array}{l}\mathrm{R} \\
\mathrm{M}\end{array}$ & $\begin{array}{l}9-18 \\
16 \pm 1.74\end{array}$ & $\begin{array}{l}11-17 \\
14 \pm 0.64\end{array}$ & $\begin{array}{l}8-11 \\
9 \pm 0.07\end{array}$ & $\begin{array}{l}12-17 \\
14 \pm 0.27\end{array}$ & $\begin{array}{l}12-16 \\
13 \pm 0.31\end{array}$ & $\begin{array}{l}11-13 \\
12 \pm 0.42\end{array}$ & $\begin{array}{l}9-13 \\
11 \pm 0.51\end{array}$ & $\begin{array}{l}5-10 \\
7 \pm 0.48\end{array}$ & $\begin{array}{l}11-18 \\
17 \pm 0.71\end{array}$ & $\begin{array}{l}20-28 \\
25 \pm 0.64\end{array}$ & 13.7 \\
\hline $\mathbf{Z n}\left(\mu \mathrm{g} . \mathrm{L}^{-1}\right)$ & $\begin{array}{l}\mathrm{R} \\
\mathrm{M}\end{array}$ & $\begin{array}{l}134-190 \\
168 \pm 5.19\end{array}$ & $\begin{array}{l}109-189 \\
158 \pm 5.14\end{array}$ & $\begin{array}{l}110-140 \\
119 \pm 4.24\end{array}$ & $\begin{array}{l}134-178 \\
150 \pm 3.72\end{array}$ & $\begin{array}{l}110-167 \\
142 \pm 4.04\end{array}$ & $\begin{array}{l}133-197 \\
163 \pm 4.26\end{array}$ & $\begin{array}{l}101-136 \\
112 \pm 3.83\end{array}$ & $\begin{array}{l}90-138 \\
102 \pm 4.36\end{array}$ & $\begin{array}{l}205-340 \\
258 \pm 4.95\end{array}$ & $\begin{array}{l}320-540 \\
375 \pm 5.29\end{array}$ & 174.7 \\
\hline $\mathbf{P b}\left(\mu \mathrm{g} . \mathrm{L}^{-1}\right)$ & $\begin{array}{l}\mathrm{R} \\
\mathrm{M}\end{array}$ & $\begin{array}{l}14-29 \\
20 \pm 0.78\end{array}$ & $\begin{array}{l}12-20 \\
16 \pm 0.88\end{array}$ & $\begin{array}{l}8-11 \\
10 \pm 0.84\end{array}$ & $\begin{array}{l}13-21 \\
15 \pm 0.92\end{array}$ & $\begin{array}{l}14-24 \\
16 \pm 0.74\end{array}$ & $\begin{array}{l}14-20 \\
16 \pm 0.81\end{array}$ & $\begin{array}{l}10-14 \\
12 \pm 0.44\end{array}$ & $\begin{array}{l}9-14 \\
11 \pm 0.49\end{array}$ & $\begin{array}{l}23-33 \\
29 \pm 0.74\end{array}$ & $\begin{array}{l}29-74 \\
51 \pm 1.20\end{array}$ & 19.4 \\
\hline $\operatorname{Mn}\left(\mu \mathrm{g} . \mathrm{L}^{-1}\right)$ & $\begin{array}{l}\mathrm{R} \\
\mathrm{M}\end{array}$ & $\begin{array}{l}41-63 \\
52 \pm 2.22\end{array}$ & $\begin{array}{l}32-70 \\
50 \pm 3.27\end{array}$ & $\begin{array}{l}38-64 \\
45 \pm 2.21\end{array}$ & $\begin{array}{l}31-57 \\
35 \pm 1.66\end{array}$ & $\begin{array}{l}10-39 \\
38 \pm 1.54\end{array}$ & $\begin{array}{l}22-91 \\
41 \pm 2.03\end{array}$ & $\begin{array}{l}38-103 \\
40 \pm 0.92\end{array}$ & $\begin{array}{l}25-59 \\
38 \pm 0.64\end{array}$ & $\begin{array}{l}39-130 \\
50 \pm 0.77\end{array}$ & $\begin{array}{l}62-164 \\
72 \pm 0.85\end{array}$ & 46.3 \\
\hline
\end{tabular}


while, the minimum cell number was recorded at Masqut-Eplis, and El-Gals (150 and 161cell X10 ${ }^{4} \mathrm{~L}^{-1}$ respectively). A more or less the same trend obtained by Dinophyta, which achieved its maximum cell number at El-Rewak ( 241 cell $\left.\times 10^{4} . \mathrm{L}^{-1}\right)$, followed by Rabaa (229 cell X10 $\left.0^{4} \mathrm{~L}^{-1}\right)$ and El-Telol (143 cell $\left.\mathrm{X} 10^{4} \cdot \mathrm{L}^{-1}\right)$. The minimum cell number of Dinophyta obtained within the middle area of the lagoon (Boughaz II, Masqut-Eplis, and El-Gals). It is worth mentioning that, if we look to each group by its percentage of abundance per the total number of cell at each site, a different perception will be obtained, as we found that the maximum percentage of Bacillariophyta (71.53\%) was recorded in Boughaz II, not in El-Rewak or Rabaa; meanwhile, the lowest percentage obtained in El-Nasser $(47.7 \%)$, Rabaa (48.35\%) and El-Rewak (49.43\%). Whereas, the lowest abundant percentage of Dinophytes $(20.5 \%)$ was recorded within Boughaz II (Table $6)$. On the whole, it is obvious that, the percentage of Bacillariophytes in eastern sites of the lake is significantly higher than in the west of the lake. However, the distribution of the Dinophyta were adverse of that, represented as percentage. Cluster analysis for the recorded algal groups reflects the relation between Bacillariophyta and Dinophyta, where they grouped in a minor sub-group with a high similarity factor (Fig.4). However, Cyanophyta, Chlorophyta, and Euglenophyta were separated in other sub-group. It is worth mentioning that Chrysophyta located single in a group with a dissimilarity factor of more than $90 \%$.

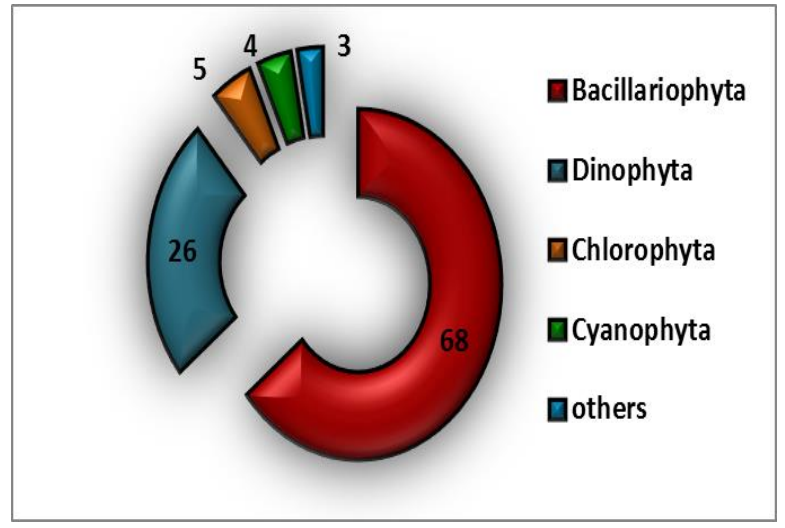

Figure (2): The Number of algal species belonging to each algal group recorded in Bardawil Lagoon.

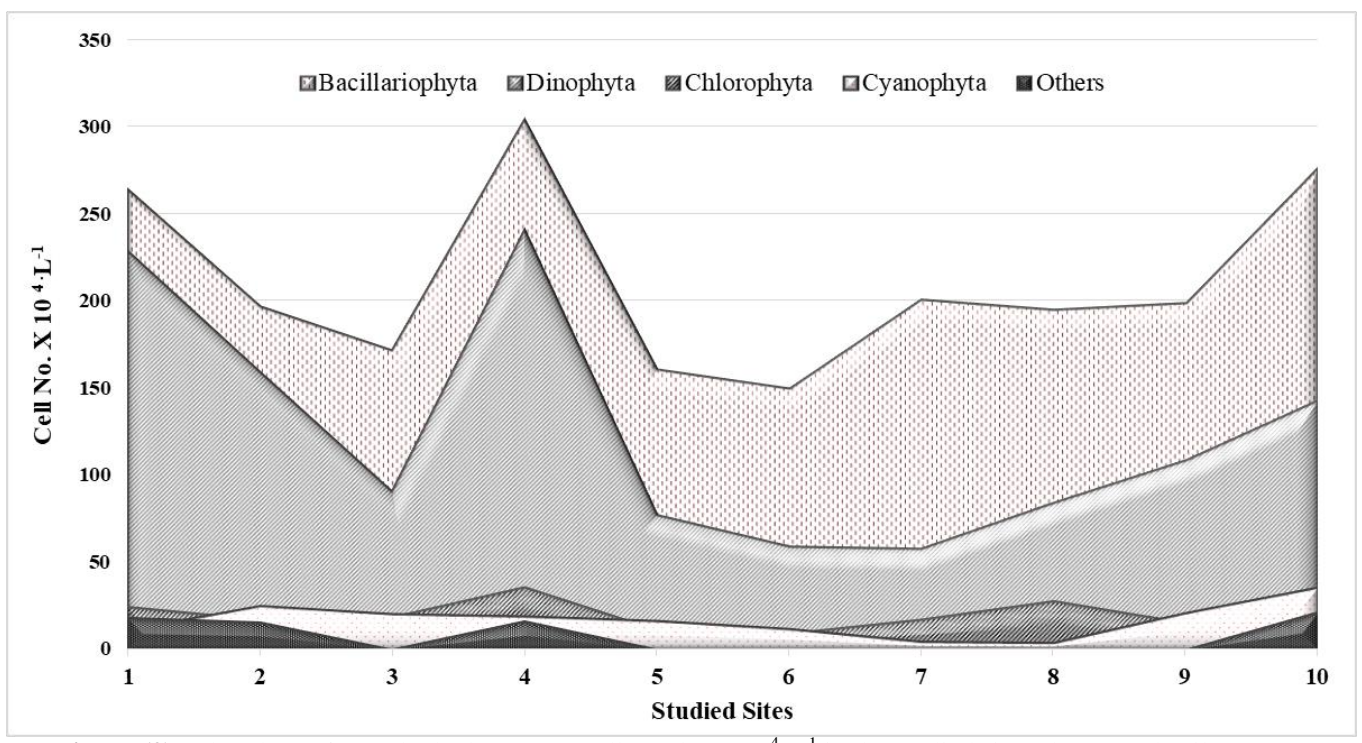

Figure (3): Abundance for each algal group as cell number $\mathrm{X} 10^{4} \mathrm{~L}^{-1}$ in the studied sites along Bardawil Lagoon.

Table (3): Pearson correlation coefficient between different water parameters measured along Bardawil Lagoon.

\begin{tabular}{|c|c|c|c|c|c|c|c|c|c|c|c|c|}
\hline Parameters & $\mathbf{p H}$ & Salinity & DO & BOD & $\mathrm{NO}_{2}$ & $\mathrm{NO}_{3}$ & $\mathbf{N H}_{4}$ & Ortho-P & $\mathrm{SiO}_{3}$ & $\mathrm{Cu}$ & $\mathbf{Z n}$ & $\mathbf{P b}$ \\
\hline pH & 1 & & & & & & & & & & & \\
\hline Salinity & 0.19 & 1 & & & & & & & & & & \\
\hline DO & -0.05 & $-0.59^{*}$ & 1 & & & & & & & & & \\
\hline BOD & 0.03 & $0.59^{*}$ & $-0.84^{* *}$ & 1 & & & & & & & & \\
\hline $\mathrm{NO}_{2}$ & -0.15 & 0.48 & $-0.67^{*}$ & $0.66^{*}$ & 1 & & & & & & & \\
\hline $\mathrm{NO}_{3}$ & $-0.59 *$ & 0.31 & -0.23 & 0.14 & 0.21 & 1 & & & & & & \\
\hline $\mathrm{NH}_{4}$ & -0.21 & $0.75^{* *}$ & $-0.79^{* *}$ & $0.86^{* *}$ & $0.62^{*}$ & $0.65^{*}$ & 1 & & & & & \\
\hline Ortho-P & 0.25 & -0.45 & $0.68^{*}$ & $-0.61^{*}$ & -0.44 & $-0.54^{*}$ & $-0.78^{* *}$ & 1 & & & & \\
\hline $\mathrm{SiO}_{3}$ & -0.34 & 0.49 & $-0.61^{*}$ & $0.67^{*}$ & $0.70^{*}$ & 0.43 & $0.59^{*}$ & $-0.55^{*}$ & 1 & & & \\
\hline $\mathrm{Cu}$ & 0.07 & $0.61^{*}$ & $-0.53^{*}$ & $0.73^{*}$ & $0.57^{*}$ & 0.28 & $0.72^{*}$ & -0.48 & $0.63^{*}$ & 1 & & \\
\hline Zn & -0.05 & $0.57^{*}$ & $-0.58^{*}$ & 0.47 & $0.51^{*}$ & 0.46 & $0.65^{*}$ & -0.43 & 0.46 & $0.81^{* *}$ & 1 & \\
\hline $\mathbf{P b}$ & 0.13 & $0.65^{*}$ & -0.43 & 0.47 & 0.3 & 0.33 & $0.69^{*}$ & -0.44 & 0.34 & $0.74^{*}$ & $0.85^{* *}$ & 1 \\
\hline Mn & -0.15 & $0.68^{*}$ & -0.29 & 0.23 & 0.46 & 0.32 & 0.43 & -0.08 & 0.34 & $0.53^{*}$ & $0.73^{*}$ & $0.66^{*}$ \\
\hline
\end{tabular}

significant correlation at $P \leq 0.05 ;{ }^{* * *}$ : highly significant correlation at $P \leq 0.001$. 
Table (4): The rate of occurrence, at a particular time during the study period represented in Frequency, for each species of the recorded algal groups recorded along Bardawil Lagoon.

\begin{tabular}{|c|c|c|c|c|c|}
\hline \multicolumn{6}{|c|}{ Algal groups } \\
\hline Representative species & Abbr..$^{\dagger}$ & Frequency & Representative species & Abbr..$^{\dagger}$ & Frequency \\
\hline \multicolumn{6}{|c|}{ Bacillariophyta } \\
\hline Achnanthes brevipes Ag. & Ac.br. & ++++ & Licmophora gracilis (Grun.) Ag. & Li.gr. & ++++ \\
\hline Achnanthes exigua Grun & Ac.ex. & ++ & Mastogloia angulata Lewis & Ma.an. & + \\
\hline Amphiprora alata (Kutz.) & Am.al. & +++ & Mastogloia braunii Grun. & Ma.br. & +++ \\
\hline Amphora coffaeiformis Ag. & Am.co & +++ & Melosira granulata (Ehr.) Ralf. & Me.gr. & ++++ \\
\hline Amphiprora paludosa W. Sm. & Am.os. & ++ & Melosira moniliformis Hust. & Me.mo. & + \\
\hline Amphora ostrearia Breb. & Am.os. & ++ & Navicula abrupta Greg. & Na.ab. & ++ \\
\hline Amphora ovalis (Kutz.) & Am.ov. & +++ & Navicula cryptocephala (Kutz.) & Na.cr. & ++ \\
\hline Asterionella japonica $\mathrm{Cl}$. & As.ja. & +++ & Navicula salinarum Grun. & Na.sa. & ++ \\
\hline Bacillaria paradoxa (Gmel.) Grun & Ba.pa. & +++ & Nitzschia hungarica Grun. & Ni.hu. & ++ \\
\hline Bacteriastrum hyalinum Cleve. & Ba.hy. & +++ & Nitzschia amphibia Grun. & Ni.am. & ++ \\
\hline Bacteriastrum delicatulum Cleve. & Ba.de. & + & Nitzschia closterium Smith & Ni.cl. & +++ \\
\hline Biddulphia mobiliensis Bailey & Bi.mo. & +++ & Nitzschia longissima (Breb.) Ralf. & Ni.lo. & ++++ \\
\hline Campylodiscus hibernicus Ehren. & Ca.hi. & ++ & Nitzschia palea (Kz.) Smith & Ni.pa. & ++ \\
\hline Campylostylus striatus Shadbolt & Ca.st. & +++ & Nitzschia sigma Smith & Ni.si. & +++ \\
\hline Chaetoceros brevis (Schutt) & Ch.br. & +++ & Nitzschia sigmoidea (Ehr.) Smith & Ni.sd. & ++++ \\
\hline Chaetoceros curvisetus Clev. & Ch.cu & +++ & Nitzschia trybionella Hantzsch. & Ni.tr. & +++ \\
\hline Chaetoceros didymus (Ehren.) & Ch.di. & ++ & Pleurosigma distortum Smith & Pl.di. & + \\
\hline Chaetoceros affinis Laud & Ch.af. & + & Rhizosolenia imbericata Cleve & Rh.im. & +++ \\
\hline Cocconeis bardawillensis Ehren. & Co.ba. & ++++ & Rhizosolenia setigera (Brigh.) & Rh.se. & +++ \\
\hline Cocconeis placentula Ehren. & Co.pl. & +++ & Skeletonema costatum (Grev.) Cl. & Sk.co. & ++ \\
\hline Cocconeis scutellum Ehren & Co.sc. & +++ & Stauroneis anceps Ehren. & St.an. & +++ \\
\hline Coscinodiscus lineatus (Ehren.) & Co.li. & +++ & Surirella clypus (Kutz.) & Su.cl. & +++ \\
\hline Cyclotella meneghiniana Kutz. & Cy.me. & +++ & Surirella striatula (Kutz.) & Su.st. & +++ \\
\hline Cyclotella planctonica Brun. & Cy.pl. & + & Synedra acus Kutz. & Sy.ac. & + \\
\hline Cyclotella ocellata Pant. & Cy.oc. & +++ & Synedra tabulata Hust. & Sy.ta. & ++++ \\
\hline Cymbella parva (W. Sm.) Cleve & Cy.pa. & ++ & Synedra ulna (Nitz.) Ehren. & Sy.ul. & ++++ \\
\hline Diatoma anceps (Ehren.) Kirch. & Di.an. & + & $\begin{array}{l}\text { Thalassionema } \\
\text { nitzschioides(Grun.) Hust. }\end{array}$ & Th.ni. & +++ \\
\hline Diploneis elliptica (Kutz.) Cleve & Di.el. & ++ & $\begin{array}{l}\text { Thalassiosira excentrica (Ehren.) } \\
\text { Cleve }\end{array}$ & Th.ex. & + \\
\hline Diploneis ovalis (Hilse.) Cleve & Di.ov. & ++ & $\begin{array}{l}\text { Thalassiosira pacifica (Grun.) } \\
\text { Hust. }\end{array}$ & Th.pa & ++++ \\
\hline Hemiaulus hauckii (Grun.) & He.ha & +++ & Thalassiothrix frauenfeldii Grun. & Th.fr. & +++ \\
\hline Leptocylindrus danicus (Celve.) & Le.da. & +++ & & & \\
\hline \multicolumn{6}{|c|}{ Dinophyta } \\
\hline Alexandrium fundyense Jorgen. & Al.fu. & ++++ & $\begin{array}{l}\text { Oxytoxum parvum (Stein) } \\
\text { Schioder }\end{array}$ & Ox.pa & +++ \\
\hline Amphidinium spheoides Wulff. & Am.sp. & ++ & Oxytoxum variabile Schiller & Ox.va. & ++ \\
\hline Ceratium egyptiacum Halim & Ce.eg. & +++ & Phalacroma argus Schiller & Ph.ar. & +++ \\
\hline Ceratium furca Ehren. & Ce.fu. & ++++ & Prorocentrum gracile Schütt & Pc.gr. & ++++ \\
\hline Ceratium tripos Nitzsch. & Ce.tr. & +++ & $\begin{array}{l}\text { Prorocentrum lima (Ehrenberg) } \\
\text { Dodge }\end{array}$ & Pc.li. & ++++ \\
\hline $\begin{array}{l}\text { Dinophysis caudata Saville-Kent } \\
\text { Dinophysis tripos Gourret }\end{array}$ & $\begin{array}{l}\text { Di.ca. } \\
\text { Di.tr. }\end{array}$ & $\begin{array}{c}+++ \\
+\end{array}$ & $\begin{array}{l}\text { Protoperidinium achromaticum Entz. } \\
\text { Protoperidinium cerasus Paulsen. }\end{array}$ & $\begin{array}{l}\text { Pr.ac. } \\
\text { Pr.ce. }\end{array}$ & $\begin{array}{c}+++ \\
++++\end{array}$ \\
\hline
\end{tabular}


Table 4: continued

\begin{tabular}{|c|c|c|c|c|c|}
\hline \multicolumn{6}{|c|}{ Algal groups } \\
\hline Representative species & Abbr. & Frequency & Representative species & Abbr. & Frequency \\
\hline Diplopsalis lenticula Bergh. & Di.le. & +++ & Protoperidinium claudicans Entz. & Pr.cl. & ++++ \\
\hline Exuviaella compressum Ostr. & Ex.co. & ++ & Protoperidinium divergens (Ehren.) & Pr.di. & +++ \\
\hline Gonyaulax apiculata (Penard) Entz. & Go.ap & ++ & Protoperidinium granii Schroed & Pr.gr. & ++++ \\
\hline Gymnodinium splendens Labour & Gy.sp. & ++ & Protoperidinium leonis (Pav.) Balech & Pr.le. & ++++ \\
\hline Gymnodinium gibberum Schilling. & Gy.gi. & +++ & Protoperidinium minutam (Ehren.) & Pr.mi. & ++++ \\
\hline Oxyphysis oxytoxoides Kafoid & Ox.ox. & ++++ & Protoperidinium steinii Jorgensen & Pr.st. & +++ \\
\hline \multicolumn{6}{|c|}{ Chlorophyta } \\
\hline Chlamydomona sp. & Ch.sp & ++ & Scenedesmus bijuga Lebour & Sc.bi & + \\
\hline Dunaliella bardawillii Halim & Du.ba & ++ & Scenedesmus sp. & Sc.sp & + \\
\hline Dunaliella salina Dunal. & Du.sa & ++ & & & \\
\hline \multicolumn{6}{|c|}{ Cyanophyta } \\
\hline $\begin{array}{l}\text { Chroococcus turgidus (Kutzing) } \\
\text { Nageli }\end{array}$ & Ch.tu. & +++ & $\begin{array}{l}\text { Oscillatoria geminata (Meneg.) } \\
\text { Gom. }\end{array}$ & Os.ge & ++ \\
\hline Oscillatoria planctonica Wolosz & Os.pl. & ++ & Spirulina subtilissima Kutz. & Sp.su. & +++ \\
\hline \multicolumn{6}{|c|}{ Euglenophyta } \\
\hline Euglena viridis Ehrenberg. & Eu.vi. & ++ & & & \\
\hline Euglena sp. & Eu.Sp & + & & & \\
\hline \multicolumn{6}{|c|}{ Chrysophyta } \\
\hline Dictyocha sp. & Di.Sp & ++ & & & \\
\hline
\end{tabular}

${ }^{\dagger}$ Abbr, Abbreviation for representative species

According to the data pertaining to both chemical and biological parameters, Detrended Correspondence Analysis (DCA) was achieved to classify the sites along Bardawil Lagoon (Figure 5). It is obvious that, Rabaa, El-Nasser, El-Rewak, El-Rodh and El-Telol were huddled together in one group "A", with high similarity between El-Rodh, and El-Telol. While Boughaz I, Boughaz II, and El- Zaranik were gathered in another group "B". On the other hand, sites El-Gals and Masqut-Eplis, were grouped in group " $\mathrm{C}$ " between "A" and "B.

Concerning the abundance index results of all the recorded species (Figures 6 and 7) at different locations along Bardawil Lagoon reflected that, the most dominant species during the investigation period were belonging to Bacillariophyta and recorded 11 species followed by Dinophyta (10 species). However, the most abundant and frequent bacillariophytes species recorded (Table 7) were Licmophora gracilis (14.81\% of the total bacillariophytes), Gyrosigma acuminatum (11.3\%), Fragilaria construens (10.1\%), Nitzschia sigmoidea (6.14 \%), Synedra tabulate (4.82\%), Synedra ulna (4.53\%), Achnanthes brevipes (3.33\%), Cocconeis bardawillensis (3.1\%), Melosira granulata (3.05\%), Nitzschia longissima (2.6\%), and Thalassiosira pacifica $(2.06 \%)$. Regarding the percentage of each algal species detected, it is of interest to refer the existence of only four bacillariophytes species including Licmophora gracilis, Gyrosigma acuminatum, Fragilaria construens, and Nitzschia sigmoidea. These type of algae were represented in high rate and reported by more than $50 \%$ of total abounded diatoms within group "A" sites (Rabaa, El-Nasser, ElRewak, El-Rodh, and El-Telol) during the time period of investigation (Table 7 and Figure 5). It is noticeable that the common bacillariophytes species (11 species) represented by more than $75 \%$ of the total diatom' cell number within all sites of group "A", and up to $93.85 \%$ within Rabaa. However, those species recorded in less percentage (than $50 \%$ ) of the rest of the sites (groups B and $\mathrm{C}$ ).

According to the abundance index results, ten species of dinophytes were the most frequent and abundant species throughout the investigation period (Figure 8 and 9). These recorded species were Protoperidinium leonis (13.73\% of the total dinophytes), Prorocentrum gracile (12.47\%), Protoperidinium granii (9.87\%), Alexandrium fundyense (6.65\%), Protoperidinium minutam $(6.64 \%)$, Ceratium furca (4.71\%), Oxyphysis oxytoxoides (4.56\%), Protoperidinium cerasus (4.23\%), Protoperidinium claudicans (3.16\%), and Prorocentrum lima (3.15\%). 


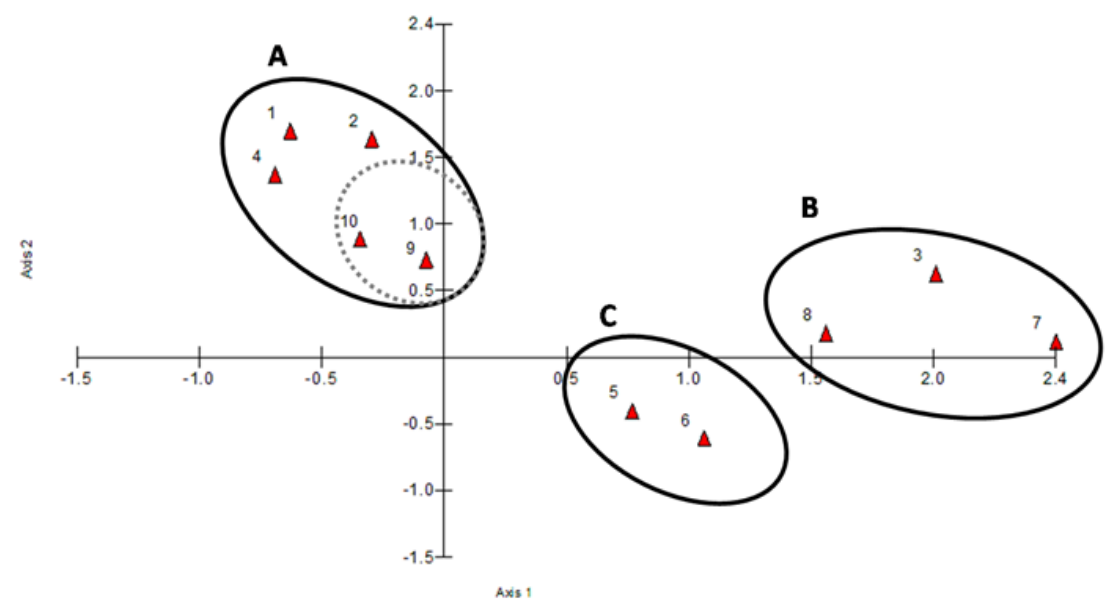

Figure (5): Detrended Correspondence Analysis (DCA) of studded sites, with respect to both chemical and biological parameters.

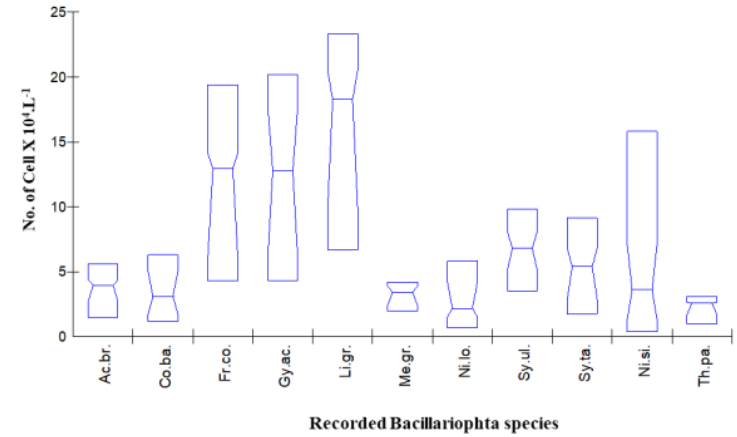

Figure (6): The abundance index of the recorded Bacillariophta species along Bardawil Lagoon.

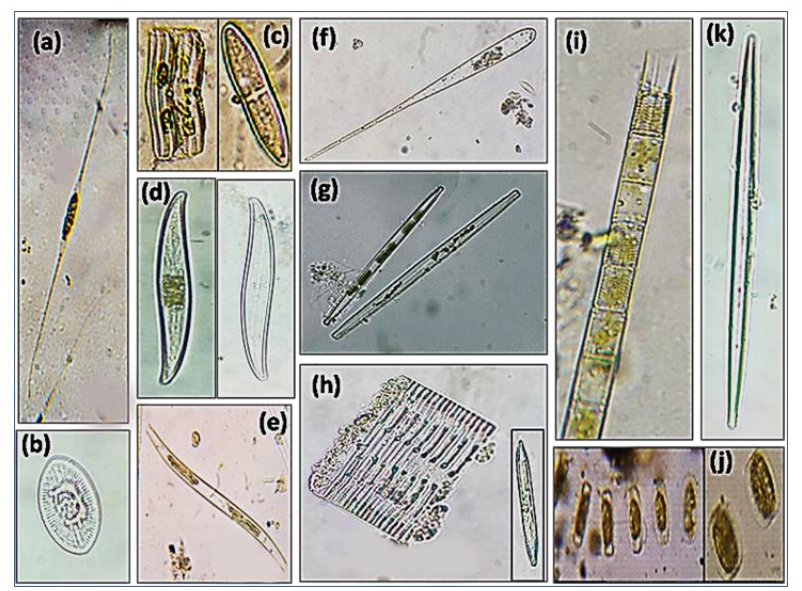

Figure (7): Light microscopy picture of common Bacillariophyta recorded, (a) Nitzschia longissima; (b) Cocconeis bardawillensis; (c) Achnanthes brevipes, (d) Gyrosigma acum-inatum; (e) Nitzschia sigmoidea; (f) Licmophora flabellate; (g) Synedra tabulate; (h) Fragilaria construens; (i) Melosira granulate; (j)Thalassiosira pacifica and (k) Synedra ulna.

The average abundance percentage of the previously mentioned common species formed more than $80 \%$ of the total abounded dinophytes within group "A" sites (Rabaa, El-Nasser, El-Rewak, El-Rodh, and El-Telol), giving it maximum value $(93.35 \%)$ at El-Rewak (Table8). Meanwhile, within the rest sites, it did not exceed $55 \%$.

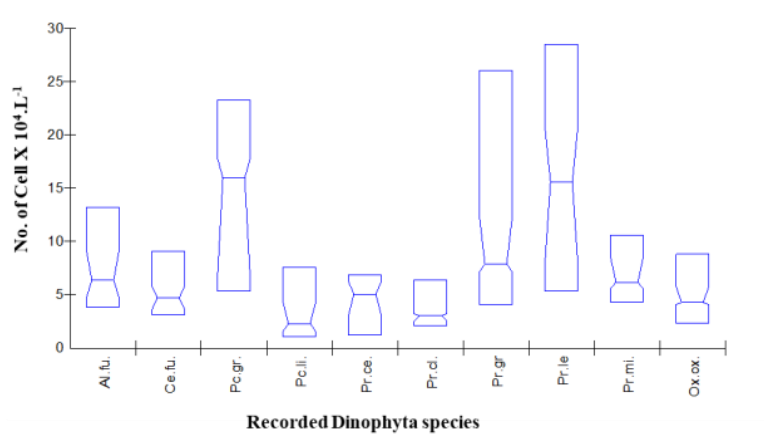

Figure (8): The abundance index of the recorded Dinophyta species along Bardawil Lagoon.

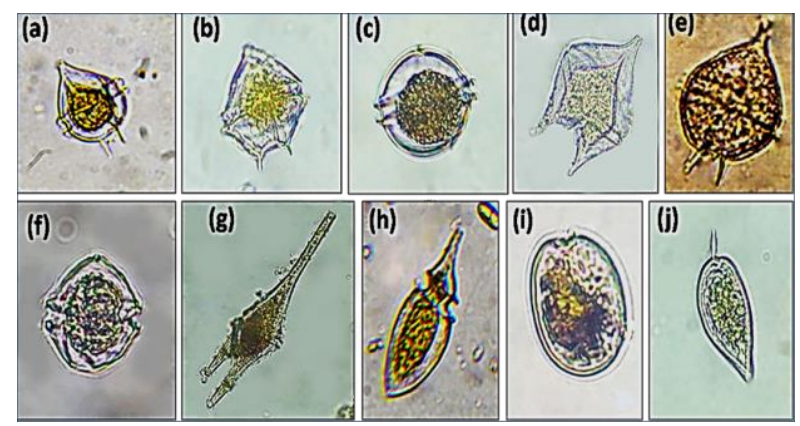

Figure (9): Light microscopy picture of common Dinophyta recorded, (a) Protoperidinium granii; (b) P. leonis; (c) $P$. minutam; (d) P. claudicans; (e) P. cerasus; (f) Alexandrium fundyense; (g) Ceratium furca; (h) Oxyphysis oxytoxoides; (i) Prorocentrum lima and (j) P. gracile

In this context, the recorded results showed that, the superior three species (Protoperidinium leonis, Prorocentrum gracile, and Protoperidinium granii) formed about $60 \%$ within Rabaa and El-Rewak. While, it represented less than $20 \%$ within Boughaz I, Boughaz II, and Masqut-Eplis.

A glance on ordination diagram produced by the Canonical Correspondence Analysis (CCA), clarify the relations between water variables and the recorded common species (Fig. 10). A remarkable relation obtained between both dissolved oxygen and orthophosphate with five species (Licmophora gracilis, 
Protoperidinium cerasus, Nitzschia longissima, Protoperidinium achromaticum and Protoperidinium claudicans). It is of interest to mention that, none of them were one of the most common species neither from Bacillariophyta nor Dinophyta. On the other side, the other recorded algal species were more related to BOD, ammonia, nitrate, nitrite, salinity, $\mathrm{pH}$, silica, and heavy metals. It is obvious from Figure. 11 that, the diversity index of all sites distributed along Bardawil Lagoon; except within El-Telol (1.9); varied from slightly polluted (3 - 4.5) to light-polluted (2 - 3). However, El-Telol classified according to the diversity index as a moderately polluted area.

Table (5): The number of species belonging to each algal group within the studied sites along Bardawil Lagoon.

\begin{tabular}{|c|c|c|c|c|c|c|c|c|c|c|c|c|}
\hline \multirow[b]{2}{*}{ Groups } & \multirow[b]{2}{*}{ Total No. of taxa } & \multicolumn{10}{|c|}{ Studied sites } & \multirow{2}{*}{ Aver. } \\
\hline & & 1 & 2 & 3 & 4 & 5 & 6 & 7 & 8 & 9 & 10 & \\
\hline Bacillariophyta & 68 & 19 & 20 & 41 & 24 & 25 & 27 & 37 & 28 & 21 & 20 & 26.2 \\
\hline Dinophyta & 26 & 14 & 14 & 17 & 14 & 13 & 13 & 16 & 15 & 9 & 8 & 13.3 \\
\hline Chlorophyta & 5 & - & - & 1 & 2 & 2 & 2 & 3 & 4 & 2 & 3 & 1.9 \\
\hline Cyanophyta & 4 & - & 1 & 2 & 1 & 2 & 2 & 3 & 2 & 1 & 2 & 1.6 \\
\hline Euglenophyta & 2 & - & - & - & 1 & 1 & - & - & - & 1 & 2 & 0.5 \\
\hline Chrysophyta & 1 & - & - & - & - & - & - & - & - & 1 & 1 & 0.2 \\
\hline Total No. of taxa & 106 & 33 & 35 & 61 & 42 & 43 & 44 & 59 & 49 & 35 & 36 & \\
\hline
\end{tabular}

Table (6): The percentage of abundance for each algal group recorded in the studied sites along Bardawil Lagoon.

\begin{tabular}{|c|c|c|c|c|c|c|c|c|c|c|c|}
\hline \multirow[b]{2}{*}{ Groups } & \multicolumn{10}{|c|}{ Studied sites } & \multirow{2}{*}{ Aver. } \\
\hline & 1 & 2 & 3 & 4 & 5 & 6 & 7 & 8 & 9 & 10 & \\
\hline Bacillariophyta & 48.35 & 47.7 & 57.14 & 49.43 & 59.63 & 65.22 & 71.53 & 62.9 & 57.51 & 57.02 & 51.94 \\
\hline Dinophyta & 42 & 38.5 & 30.23 & 39.19 & 28.52 & 25.65 & 20.5 & 27.1 & 31.5 & 29.55 & 28.31 \\
\hline Chlorophyta & 4.4 & 4.12 & 5.98 & 5.69 & 4.07 & 3.91 & 6.05 & 8.71 & 4.34 & 1.86 & 4.72 \\
\hline Cyanophyta & 2.01 & 6.05 & 6.64 & 3.09 & 5.93 & 5.22 & 1.78 & 1.29 & 6.07 & 7.23 & 3.81 \\
\hline others & 3.3 & 3.63 & 0 & 2.6 & 1.85 & 0 & 0 & 0 & 0.58 & 4.34 & 1.19 \\
\hline
\end{tabular}

Table (7): The percentage of abundance for the common Bacillariophyta species recorded in the studied sites along Bardawil Lagoon.

\begin{tabular}{|c|c|c|c|c|c|c|c|c|c|c|c|}
\hline \multirow{2}{*}{ Bacillariophyta species } & \multicolumn{10}{|c|}{ Studied sites } & \multirow[b]{2}{*}{ Aver. } \\
\hline & 1 & 2 & 3 & 4 & 5 & 6 & 7 & 8 & 9 & 10 & \\
\hline Achnanthes brevipes & 5.62 & 3.97 & 0.94 & 4.39 & 2.1 & 2.9 & 1.5 & 2.7 & 4.3 & 4.9 & 3.3 \\
\hline Cocconeis bardawillensis & 5.1 & 3.8 & 2.3 & 1.7 & 6.3 & 0.3 & 1.2 & 1.6 & 3.1 & 5.6 & 3.1 \\
\hline Fragilaria construens & 14.12 & 12.94 & 6.21 & 19.39 & 6.81 & 4.3 & 3.38 & 6.21 & 13.2 & 14.47 & $10 . .1$ \\
\hline Gyrosigma acuminatum & 20.15 & 17.7 & 4.28 & 18.16 & 6.1 & 5.9 & 3.9 & 7.34 & 12.75 & 16.7 & 11.3 \\
\hline Licmophora gracilis & 23.31 & 20.51 & 10.36 & 20.14 & 9.98 & 9.58 & 6.68 & 6.3 & 18.26 & 22.95 & 14.81 \\
\hline Melosira granulata & 4.18 & 3.9 & 1.2 & 4.22 & 3.1 & 2.4 & 2 & 2.4 & 3.42 & 3.7 & 3.05 \\
\hline Nitzschia longissima & 1.52 & 0.7 & 1.9 & 2.73 & 0.5 & 0.8 & 4.2 & 5.83 & 5.61 & 2.2 & 2.6 \\
\hline Nitzschia sigmoidea & 7.33 & 6.11 & 3.54 & 9.32 & 1.7 & 6.8 & 3.51 & 5.09 & 8.15 & 9.8 & 6.14 \\
\hline Synedra tabulata & 9.13 & 6.08 & 1.75 & 8.36 & 2.4 & 1.7 & 2.86 & 3.65 & 5.41 & 6.83 & 4.82 \\
\hline Synedra ulna & 0.8 & 1.38 & 15.8 & 0.37 & 3.62 & 7.13 & 9.7 & 4.89 & 0.33 & 1.29 & 4.53 \\
\hline Thalassiosira pacifica & 2.59 & 1.76 & 0.98 & 3.1 & 0.91 & 2.7 & 1.7 & 1.6 & 2.67 & 2.6 & 2.06 \\
\hline$\%$ of Total Bacillariophyta & 93.85 & 78.85 & 49.26 & 91.88 & 43.52 & 44.51 & 40.63 & 47.61 & 77.2 & 91.04 & \\
\hline
\end{tabular}




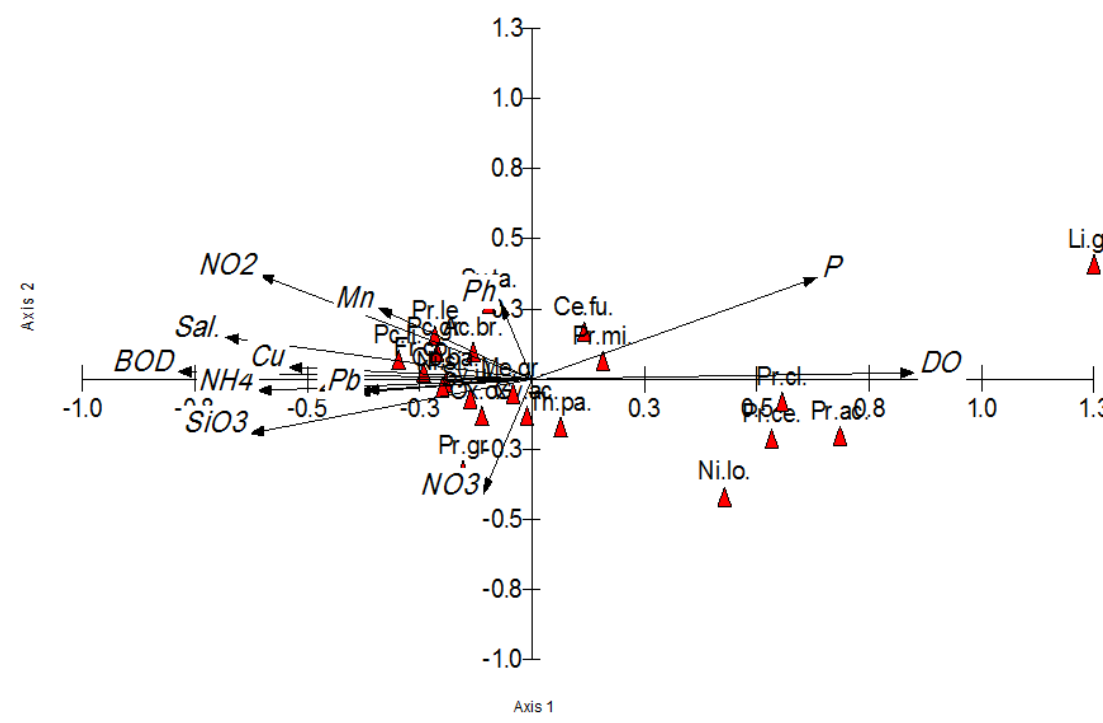

Figure (10): Canonical Correspondence Analysis (CCA) joint plot ordination diagram for common species (points) with water variables (arrows) along Bardawil Lagoon. (The species names are abbreviated to the first letter from the name of genus and species. For full names see table: 4 ).

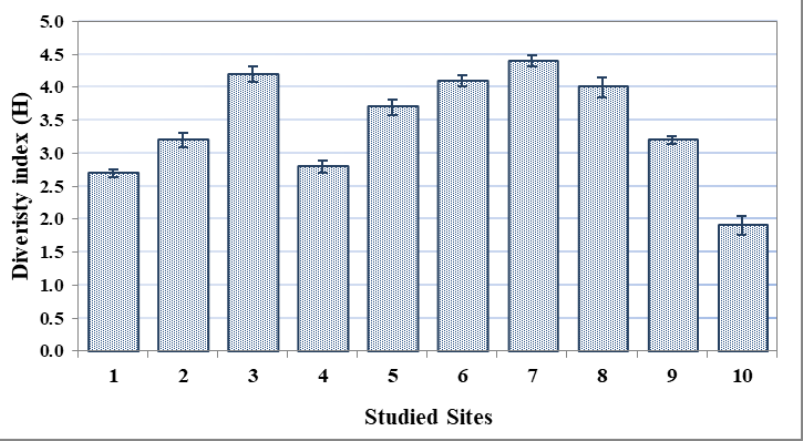

Figure (11): The average of diversity index along Bardawil Lagoon' sites.

\section{DISCUSSION}

Bardawil Lagoon considered the largest hypersaline Mediterranean coastal lagoons, with a surface area of $685 \mathrm{~km} 2$, followed by Spanish Mediterranean Lagoon, Mar Menor $\left(135 \mathrm{~km}^{2}\right)$. On the other hand, it has global renown for high-quality fishes production, besides that, it also considered a good habitat for resting migratory birds, therefore, both the physicochemical and biological composition must gain the attention. The average value of hydrogen ion concentration $(\mathrm{pH})$ in the studied area fluctuated within a narrow range on the alkaline side (from 7.6 to 8.8), the results which agree with that recorded by Khalil et al., (2013) where it ranged between 7.1-and 8.8. Siliem (1989) in his studies on the chemical conditions in Bardawil Lagoon reported that $\mathrm{pH}$ varied from 7.5 to 8.76 with an annual average of 8.16 . It is notable that, $\mathrm{pH}$ values showed a slight variation among different stations. The salinity of Bardawil Lagoon is higher than that of the open Mediterranean Sea (Khalil and Shaltout, 2006) due to the low rainfall rate $(80-100 \mathrm{~mm} /$ year $)$ and the high evaporation rate $(1460 \mathrm{~mm} / \mathrm{year})$. The salinity of the lagoon showed miscellaneous temporal and spatial behavior giving its maximum value $\left(72.7\right.$ g.L $\left.\mathrm{L}^{-1}\right)$ within Rabaa site and the minimum within Boughaz II (37.5 g.L $\mathrm{L}^{-1}$ ) followed by Boughaz I (37.9 g.L $\left.{ }^{-1}\right)$. The obtained results in the present study clarify that, the decrease in salinity was relatively related to the distance from the two artificial inlets with the sea (Boughaz I and II) at which the lagoon exchange water with the Mediterranean Sea. Therefore, the values of salinity in the lagoon depend upon the state of the inlets; Where during the opening period (after dredging) the salinity was lower than its recorded values during partial closure of the inlets by sand (Ali et al., 2006; Mehanna, 2014). The disturbing problem is that those artificial inlets have a natural tendency to be closed completely or partially by sand carried by waves and the current along the coast, at the same time, the tidal flow is too weak to keep them open. Accordingly, the present study revealed that, the maximum salinity was recorded in the eastern and western edges of the lagoon. Therefore, the competent authorities should pay more attention to the state of the lagoon inlets and must incite the continuous dredging during the year to remove the accumulated sand

Dissolved oxygen appraise as the key factor of life in aquatic habitats. It is essential to the metabolic activities of most aquatic organisms (Mofeed and Abdel-Aal, 2015). On the other hand, it required for transformation reactions (oxidation, nitrification, and decomposition) of all the chemical compounds in the water (Touliabah et al., 2002; Mofeed and Deyab, 2015). The average of dissolved oxygen during the investigation period fluctuated from 5.4 to $8.5 \mathrm{mg} . \mathrm{L}^{-1}$, which means that Bardawil Lagoon can be considered as a well-oxygenated ecosystem because it usually contains concentrations of DO above the minimum WHO standard $\left(5 \mathrm{mg} . \mathrm{L}^{-1}\right)$ in water quality assessment (Nkwo et al., 2010). Negm et al., (2019) reported that, 
the recorded dissolved oxygen in Bardawil Lagoon showed a distinct high values compared with the other four northern Egyptian Lagoons. A more or less the same results were recorded by Fouda et al., (1985); Khalil et al., (2013); Ali et al., (2006); Mehanna (2014); El-Sheekh et al., (2019). However, the biological oxygen demand in all the studded sits varied from 1.3 to $6.7 \mathrm{mg} . \mathrm{L}^{-1}$, giving its maximum within ElRewak. This result is compatible with the biological results, where the maximum phytoplankton abundance was also recorded at El-Rewak.

Distribution of nutrients in coastal water is affected by regional conditions such as tidal incursion, rainfall rate, inflow of freshwater and biological activities such as uptake by phytoplankton beside the anthropogenic activities (Satpathy et al., 2010). Khalil and Shaltout (2006) reported that, generally concentrations of dissolved inorganic nitrogen were low, with a noticeable increase near the inlets due to exchanging water with the sea. However, the maximum values of nitrite, nitrate and ammonia were recorded within ElTelol (9.8, 58.6 and $96 \mu \mathrm{g} . \mathrm{L}^{-1}$ respectively). This superiority of El-Telol may be attributed to the human activities of the fishermen community in that area. The obtained annual average of nitrate, nitrite, and ammonia were 43.32, 6.53 and $51.9 \mu$ g.. ${ }^{-1}$ respectively. In a previous study by El-Kassas et al., (2016), the annual averages of nitrate, nitrite, and ammonia were $32.25,2.25$ and $60.25 \mu \mathrm{g} . \mathrm{L}^{-1}$ respectively, while Khalil et al., (2013), demonstrated that, the annual average value of nitrite, nitrate and ammonia respectively were $4.5,42$, and $48 \mu \mathrm{g} . \mathrm{L}^{-1}$, furthermore, he reported that, ammonia was the main source of inorganic nitrogen .

Phosphate is the most essential nutrient that can govern the production of phytoplankton in coastal ecosystems, and consequently, the variation in phytoplankton depends upon the phosphate content (Shaaban-Dessouki et al., 2004). Ortho-phosphate showed its minimum regional mean at El-Telol (32 $\left.\mu \mathrm{g} . \mathrm{L}^{-1}\right)$ however, the maximum values were recorded within Boughaz I (48 $\left.\mu \mathrm{g} . \mathrm{L}^{-1}\right)$, followed by Boughaz II (46 $\mu \mathrm{g} . \mathrm{L}^{-1}$ ) and it gradually decreased southward during the study period with an annual average of 39.7 $\mu \mathrm{g} . \mathrm{L}^{-1}$. This phenomenon indicated that phosphates were passed from the seawater into the lagoon and then often because of the high salinity of lagoon water than the seawater, the inorganic phosphorus precipitated in sediment. El-Kassas et al., (2016) reported that, the average of phosphate concentrations was $2.44 \mu \mathrm{g} . \mathrm{L}^{-1}$, while Khalil et al., (2013) indicated that, it reached 35 $\mu \mathrm{g} . \mathrm{L}^{-1}$. Concerning silicate revealed that it had a regional variation fluctuated from $69 \mu \mathrm{g} . \mathrm{L}^{-1}$ at El-Galas to $133 \mu \mathrm{g} . \mathrm{L}^{-1}$ at El-Telol. In coastal water, the spatial variation of silicate affected by several factors; the biological uptake by phytoplankton (bacillariophytes and silicoflagellates), the proportional mixing of seawater with fresh-water or even the adsorption of reactive silicate by suspended sedimentary particles (Satpathy et al., 2010). The estimated heavy metals showed the maximum concentrations within El-Telol, while El-Rodh comes in the second position with a significant gap. These high values of heavy metals at the El-Telol attributed receiving the tailings of the fishermen' boats. The minimum values always recorded within El- Zaranik. The result, which was in accordance that demonstrated by Ali et al., (2006) in his study on water quality of Bardawil lagoon.

Generally, the environmental variables not only control phytoplankton abundance but also significantly influence its community composition (Blanco et al., 2008), especially in such ecologically peculiar of Bardawil Lagoon with its shallow and semi-enclosed water. Therefore, it had specific structural and functional characteristics resulting from their location. The recorded data during the entire period of investigation revealed that there were remarkable spatial variations in both qualitative and quantitative phytoplankton composition reflecting the environmental factors, especially salinity, dissolved oxygen, and nutrient availability. Therefore, the characteri-zation of phytoplankton communities becomes essential in recognizing the quality of ecosystems (Cermeno et al., 2011). A relatively high total number of taxa (106 taxa) recorded during the study period, reflecting rich phytoplankton communities in the Lagoon. The maximum number of species was recorded near the opening of the artificial inlets (Boughaz I and Boughaz II), this may be attributed to exchanges water currents between the lagoon and the Mediterranean Sea with its planktonic species and lower water salinity. While the minimum numbers of taxa were recorded on the eastern and western sides of the lagoon, due to the high salinity that may impede the growth of some species. In all sites, tyrant dominion of the bacillariophytes in terms of the number of species over the other algal groups, followed by dinophytes. A more or less the same trend of dominance in taxa number obtained by El-Kassas et al., (2016); Khalil et al., (2013) and Ali et al., (2006) Zalat et al., (2019) ElSheekh et al., (2019).

\section{CONCLUSION}

Referring to the importance of Bardawil Lagoon from both environmental and economic points of view, as a source of the high-quality export fish, as well due to its distinguished geographical location and containing a natural protected area (Zaranik Protected Area) for migratory organisms including some endangered species, it is imperative that we have a role in monitoring the environmental situation and dealing with it in a sophisticated way, especially as the urban extension and human activities extends to this area quickly. This study monitored the changes in the chemical characteristic of water, as well as the abundance and distribution in the phytoplankton composition which showed the same tend of dominancy as algal groups but not as species composition from what was observed in previous studies due to the increase in unauthorized human activities. Meanwhile, the supremacy of Bacillariophyta and Dinophyta occurrence was recorded along the study period despite the hyper-saline nature of the 
lagoon. This can be explained that this condition may be supportive for the flourishing of such species. While the recorded results at the level of species showed that the common species that prevailed throughout the study were other than the dominated species in previous studies, such as Licmophora gracilis, Gyrosigma acuminatum, Fragilaria construens, Nitzschia sigmoidea, Protoperidinium leonis, Prorocentrum gracile, and Protoperidinium granii. This may threaten the balance of the ecosystem in the lake, which will directly be reflected in its economic productivity. Biodiversity increased near the two inlets (Boughaz I and II), at which the water in the Lagoon renew and refresh. Hence, regular dredging to the sand accumulated in the two inlets by the action of water current and tide became an urgent necessity. Therefore, this study recommends further continuous follow-up by ecological assessment and monitoring studies of Bardawil Lagoon. Where one of the most important priorities of science and society is to follow the environmental status of natural reserves that have become limited in the world due to the spread of pollution in various forms all over the world.

\section{ACKNOWLEDGMENT}

Author expresses her appreciation to all hydrobiology lab staff and fishermen for their help during the samples collection.

\section{REFERENCES}

AJAYKUMAR, A.V., S.A.L. HASHIMI, AND N. HILAL. 2008. Investigation of kinetics and mechanism involved in the biosorption of heavy metals on activated sludge. Int. J. Green Energy 5: 313-321.

ALI, H.H.M., M. F. SAYED, AND M. E. GOHER. 2006. Studies on water quality and some heavy metals in hypersaline Mediterranean lagoon (Bardawil Lagoon, Egypt). Egypt. J. Aquat. Biol. and Fish., Vol. 10, No.4:45 - 64.

AMERICAN PUBLIC HEALTH ASSOCIATION (APHA). 1989. Standard methods for the examination of water and wastewater pp 62617 th ed, New York.

ANONYMOUS. 1993. SPSS program for Windows. Base system user's Guide Release 5.0 SPSS INC.

BAKER, P. 1991. Identification of common cyanobacteria. Part I - Nostocales, Urban Water Research Association of Australia, Research reportno. 29, June 1991, Australian Centre for WaterTreatment and Water Quality Research, Melbourneand Metropolitan Board of Works, Melbourne.

BARBARY, M. S. A., AND G. M. EL-SHABRAWY. 2004. Community structure and biochemical parameters of annelid worms in Bardawil Lagoon. J. Egypt. Ger. Soc. Zool., 45(D):469-492.

BARNES, H., AND A. R. FOLKARD. 1951. The determination of nitrites. Analyst (London) 76. 599 $-603$.

BLANCO, A.C., K.NADAOKA, AND T. YAMAMOTO. 2008. Planktonic and benthic microalgal community composition as indicators of terrestrial influence on a fringing reef in Ishigaki Island, Southwest Japan. Marine Environmental Research 66, 520- 535.

BREMNER, J.M., AND K. SHAW.1955. Determination of ammonia and nitrate in soil. 1. Agr. Sci. 46, 320-328.

CERMENO, P., J. LEE, K.WYMAN, AND O.SCHOFIELD. 2011. Competitive dynamics in two species of marine phytoplankton under non-equilibrium conditions. Marine ecology progress series. Vol. 429: 19-28.

DORGHAM, M.M., N.E. Abdel-Aziz, K.Z. El-Deeb, and M.A.Okbah. 2004. Eutrophication problem in the Western Harbour of Alexandria, Egypt," Oceanologia, 46(1),pp. 1-20.

EL-AIATT A. A.O., SHALlOOF, K.A.SH., AND BER, M.M. 2019. Bio-economic studies on the catch of Bardawil Lagoon, North Sinai,Egypt. Egyptian Journal of Aquatic Research, 45:59-65.

EL-KASSAS, H. Y., M.N. ZEIN ALABEDIN, AND S. M. GHARIB. 2016. Study of phytoplankton in a natural hypersaline lagoon in a desert area (Bardawil Lagoon in Northern Sinai, Egypt). Rend. Fis. Acc. Lincei (2016) 27:483-493.

EL-SHEEKH, M., ALI E., AND EL-KASSAS, H. 2019, Phytoplankton ecology along the Egyptian northern lakes: status, pressures and impacts. Hdb Env. Chem, 71: 133-172.

EL-SHERIF, Z.M., M.Z. NASSAR, AND M.A. FAHMY. 2010. Phytoplankton distribution in the southeastern Mediterranean Sea (Egyptian waters) in summer and winter 2005.Egypt. J. Aquat. Res., 36 (4), pp. 609-621.

FOGED, N. 1978. Diatoms in eastern Australia', Bibliotheca Phycologica, 47, pp. 1-255.

FOUDA, M.M ., M.K. WANES, AND M.A. SALEH. 1985. Ecology of Bradawil lagoon. A report to the Oil Pollution Res. Unit, Pembroke, U.K. for B.P Petroleum Ltd. Egypt.

GAFRD .2012. (General Authority for Fish Resources Development). Fisheries Statistics Year Book 2012, Cairo, Egypt, GAFRD Press., 300 pp.

GHARIB, S.M., AND M.M. DORGHAM. 2006. Eutrophication stress on phytoplankton community in the Western Harbour of Alexandria, Egypt. Int $\mathbf{J}$ Ocean Oceanogr 1:261-273.

HARTIKAINEN, H. 1979. Phosphorus and its reactions in terrestrial soils and lake sediments. J. Scient. Agric. Soc. Finl., 53. 16 - 26.

HENDEY, N.I .1964. An introductory account of the smaller algae of British coastal waters. Part 5: Bacillariophyceae (Diatoms). Her Majesty ${ }^{\text {ee }}$ Stationery Office, London.

KAMPSHAKE, L.J., S. A. HANNAH, AND J. M. COHEN. 1967. Automated analysis for nitrate by hydrazine reduction. Water Resour. Res. 1. 205- 216.

KHALIL, M.T., A.A. SAAD, M. R. FISHAR, AND T.Z. BEDIR. 2013. Ecological Studies on Macrobenthic Invertebrates of Bardawil Wetland, Egypt. World Environment, 3(1): 1-8. 
KHALIL, M.T., AND K.H. SHALTOUT. 2006. Lake Bardawil and Zaranik Protected Area. Publication of National Biodiversity Unit. No. 15.

KONSOWA, A. 2007. Phytoplankton evolution in a shallow hypertrophic saline lake. Azhar Journal of Pharmaceutical Sciences, 32: 109 - 122.

KRAUSSE, G. L., C. L. SCHELSKE, AND C. O. DAVIS. 1983. Comparison of three wet alkaline methods of digestion of biogenic silica. Freshwat. Biol., 13. 73 - 81.

LEGENDRE, P., AND L. LEGENDRE.1998. Numerical ecology. Elsevier. Developments in environmental modelling, 3. Elsevier Scientific Publ. Co., Amsterdam Netherlands 9: 419 p.

MEHANNA, S.F. 2014. Reproductive dynamics of the common sole Soleasolea (Linnaeus, 1758) from Bardawil lagoon, North Sinai, Egypt. Conference on International Research on Food Security, Natural Resource, September 17-19, 2014, Tropentag, Prague, Czech Republic.

MOFEED, J. 2015a. Effect of different concentrations of polluted water on growth and physiological parameters of two green algae Scenedesmus obliquus and Cosmarium leave. J. Environmental Science, vol, 44(1): 171-184.

MOFEED, J. 2015b. Effects of salinity and light intensity on production of $\beta$-carotene and glycerol from the halotolerant alga Dunaliella salina (Chlorophyta) isolated from Zaranik nature reserve, North Sinai, (Egypt). Egypt. J. Exp. Biol. (Bot.), 11 (1): 21- 29.

MOFEED, J., AND E. I.ABDEL-AAL. 2015. Effect of phenol on some antioxidant enzymes in the marine microalga Dunaliella salina. J. Environmental Science,vol, 44(1): 185 - 196.

MOFEED, J., AND M. A. DEYAB. 2015. Monitoring for the abundance and distribution of macro algae along Suez Canal, Egypt. CATRINA, 11 (1): 81-91.

MOFEED, J., AND Y. Y. MOSLEH. 2013. Toxic responses and antioxidative enzymes activity of Scenedesmus obliquus exposed to fenhexamid and atrazine, alone and in mixture. Ecotoxicology and Environmental Safety 95, 234 - 240.

NEGM, A. M., M. ALI BEK, AND S.ABDELFATTAH. 2019. Egyptian Coastal Lakes and Wetlands (Part I)Characteristics and Hydrodynamics Editors-in-Chief: Damia Barcelo' • Andrey G. Kostianoy Volume 71.

NKWO, J.A., C. ONYEMAI, AND J.K. IGBO. 2010. Wet season spatial occurrence of phytoplankton and zooplankton in Lagos Lagoon, Nigeria. Sci World J 5:7-14.

OVIATT, C. A. 2004. The changing ecology of temperatecoastal waters during a warming trend. Estuaries27: 895-904. doi:10.1007/BF02803416.

PATRICK, R., AND C.W. REIMER. 1966. The diatoms of the USA, Vol. I, Monographs of the Academy Natural Science, Philadelphia.

PISANTY, S. 1980. The fishery and management of the hypersaline Lagoon of Bardawil. GFCM Stud. Rev., 58: 35-73.
PRESCOTT GW .1982. Algae of the western great lakes area with an illustrated key to the genera of desmids and freshwater diatoms. Koeningstein: Otto Koeltz Science Publishers

PRESCOTT, G.W. 1962. Algae of the western Great Lakes area Brown, W. C., Dubuque (IOWA) pp. 977.

PRESCOTT, G.W. 1969. The algae, A Review, Nelson and Sons Led. New York, .416.

SATPATHY, K. K., A. K. MOHANTY, U. NATESAN, M. V. R. PRASAD, AND S. K. SARKAR. 2010. Seasonal variation in physico-chemical properties of coastal waters of Kalpakkam, East coast of India with special emphasis on nutrients. Environ. Monit. Assess., 164, p. 153-171.

SAWANT, S.S., AND M. MADHUPRATAP. 1996. Composition of phytoplankton in the Arabian Sea, Curr Sci, 71 869-873.

SHAABAN-DESSOUKI, S.A., M.A. DEYAB, AND J. MOFEED. 2004. Phycological assessment of water quality of river nil delta Egypt, Jour. of phycology (5) $19-34$.

SHAMS EL DIN G.N., AND A.M. ABDEL HALIM. 2008. Changes in phytoplankton community structure at three touristic sites at western Alexandria Beach, Egypt. J Aquat Biol Fish 12:85118.

SHAMS EL DIN, G.N., AND E.M. ABO EL KHAIR. 2014. Dorgham M., M. Phytoplankton community in the Egyptian Mediterranean coastal waters. Indian Journal of Geo-Marine Sciences. Vol. 43(10)pp. 1981-1988.

SHANNON, C.E., AND W. WEAVER.1949. The mathematical theory of communication. University of Ilinois Press, Urbana. 125 pp.

SILIEM, T.A.A. 1989. The chemical conditions in Bardawil Lagoon. IV-Nutrient salts. Bull. Nat. Inst. Oceanogr. \& Fish. ARE., 15: 217 - 228.

STARMACH, K. 1983. Euglenophyta- Egleniny.Flora Slodkowodna Polski, Tom 3:1-563.

STOMACH, K. 1968. Chrysophyceae- Zlotowiciowce oraz Flora Slodkowod. Pol.,5: 1-598.

TER-BRAAK, C. J. F. 1988. CANOCO: A FORTRAN program version 2.1 for (partial) (detrended) (canonical) Correspondence Analysis, Principal Component Analysis and Redundancy Analysis. Agricultural. Mathematics Group, Report LWA-8802, Wageningen. $95 \mathrm{pp}$.

TOULIABAH, H., H.M. SHFIK, M.M. GAB-ALLAH, AND W.D. TAYLOR. 2002. Phytoplankton and some abiotic features of El-Bardawil Lake, Sinai Egypt. Afr. J. Aqu. Sci., 27: 97 - 105.

YAMAGISHI, T. 1992. Plankton algae in Taiwan (Formosa):1-252- Uchida Rokakuho, Tokyo.

ZALAT A. A., EL-SHEEKH M. M. AND GABALLA M. 2019. Distribution Pattern of Diatom Flora in the Surface Sediments of Bardawil Lagoon (North Sinai), Egypt. International Journal of Marine Sciences, 10.1007/s41208-019-00160-4. 


\title{
رصد وفرة العوالق النباتية وتوزيعها على طول بحيرة بردويل الثاطئية في شمال سيناء ، مصر (نهج التحليل متعدد المتغيرات)
}

\author{
جيلان مفيد \\ قسم البيئة المائية ، كلية الثروة السمكية، جامعة السويس،السويس، مصر \\ الملخص العربـــي
}

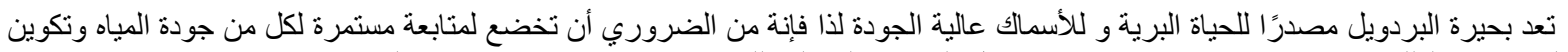

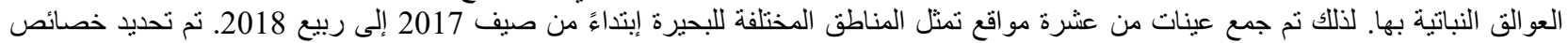

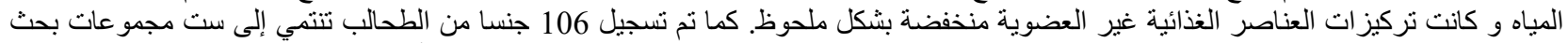

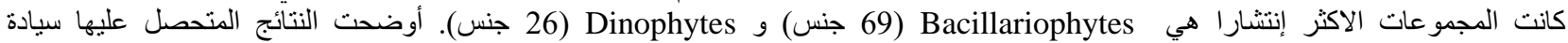
فacillariophyta

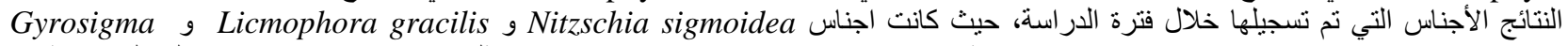

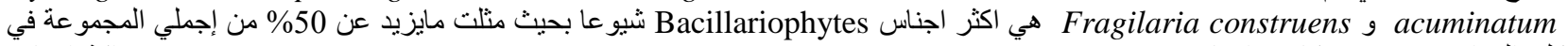

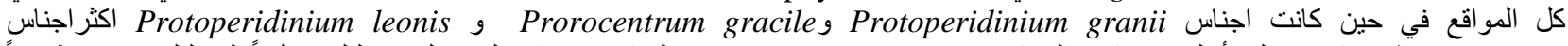

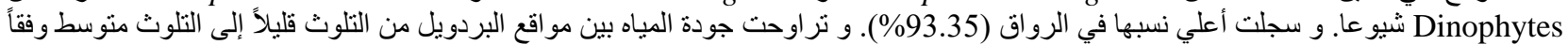

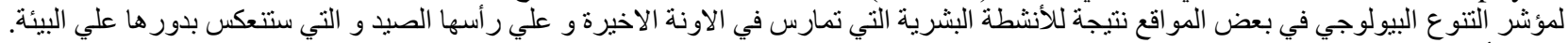

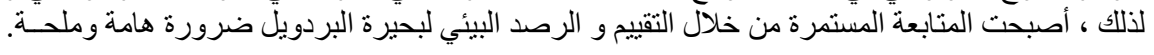

